The Astrophysical Journal, 674:447-450, 2008 February 10

(C) 2008. The American Astronomical Society. All rights reserved. Printed in U.S.A.

\title{
THE ENVIRONMENT OF M85 OPTICAL TRANSIENT 2006-1: CONSTRAINTS ON THE PROGENITOR AGE AND MASS
}

\author{
E. O. Ofek, ${ }^{1}$ S. R. Kulkarni, ${ }^{1}$ A. Rau,${ }^{1}$ S. B. Cenko, ${ }^{1}$ E. W. Peng, ${ }^{2}$ J. P. Blakeslee, ${ }^{3}$ P. Côté ${ }^{2}$ L. Ferrarese, ${ }^{2}$ \\ A. Jordán, ${ }^{4}$ S. Mei, ${ }^{5}$ T. Puzia, ${ }^{2}$ L. D. Bradley, ${ }^{6}$ D. Magee, ${ }^{7}$ and R. Bouwens ${ }^{7}$ \\ Received 2007 July 12; accepted 2007 October 9
}

\begin{abstract}
M85 Optical Transient 2006-1 (M85 OT 2006-1) is the most luminous member of the small family of V838 Mon-like objects, whose nature is still a mystery. This event took place in the Virgo Cluster of galaxies and peaked at an absolute magnitude of $M_{I} \approx-13$. Here we present Hubble Space Telescope images of M85 OT 2006-1 and its environment, taken before and after the eruption, along with a spectrum of the host galaxy at the transient location. We find that the progenitor of M85 OT 2006-1 was not associated with any star-forming region. The $g$-and $z$-band absolute magnitudes of the progenitor were fainter than about -4 and -6 mag, respectively. Therefore, we can set a lower limit of $\sim 50 \mathrm{Myr}$ on the age of the youngest stars at the location of the progenitor that corresponds to a mass of $<7 M_{\odot}$. Previously published line indices suggest that M85 has a mean stellar age of $1.6 \pm 0.3 \mathrm{Gyr}$. If this mean age is representative of the progenitor of M85 OT 2006-1, then we can further constrain its mass to be less than $2 M_{\odot}$. We compare the energetics and mass limit derived for the M85 OT 2006-1 progenitor with those expected from a simple model of violent stellar mergers. Combined with further modeling, these new clues may ultimately reveal the true nature of these puzzling events.
\end{abstract}

Subject headings: stars: individual (M85 OT 2006-1, V838 Mon, M31 RV, V4332 Sgr)

\section{INTRODUCTION}

M85 optical transient 2006-1 (M85 OT 2006-1 [=J122523.82+ 181056.2]) was discovered on 2006 January 6 by the Lick Observatory supernova search team (Filippenko et al. $2001^{8}$ ) as a faint, $V \sim 19.3$ mag transient in the galaxy M85 (NGC 4382), which is at a distance of $17.8 \mathrm{Mpc}$ (Mei et al. 2007). Subsequent spectroscopy, as well as visible light and infrared (IR) photometry, presented in Kulkarni et al. (2007), showed that M85 OT 2006-1 has a recession velocity of $880 \pm 130 \mathrm{~km} \mathrm{~s}^{-1}$, and is therefore associated with M85. Moreover, we have shown that the temporal and spectral properties of this object are unlike those of supernovae, novae, or luminous blue variables.

M85 OT 2006-1 peaked at absolute $I$-band magnitude of about -13 . The light curve settled into a $\sim 60$ day plateau, followed by a decrease in bolometric luminosity during which the blackbody emission peak shifted toward near-IR wavelengths. The early spectrum of M85 OT 2006-1, obtained 6 weeks after discovery, resembles that of a $\sim 4600 \mathrm{~K}$ blackbody, with $\mathrm{H} \alpha$ and $\mathrm{H} \beta$ narrow emission lines $\left(\mathrm{FWHM} \sim 350 \mathrm{~km} \mathrm{~s}^{-1}\right.$ ), along with several other unidentified emission lines.

Spitzer Space Telescope IR observations obtained about 6 months after the discovery revealed a $\sim 1000 \mathrm{~K}$ blackbody spectral energy distribution ( Rau et al. 2007).

\footnotetext{
${ }^{1}$ Division of Physics, Mathematics and Astronomy, California Institute of Technology, Pasadena, CA 91125.

${ }^{2}$ Herzberg Institute of Astrophysics, Dominion Astrophysical Observatory, Victoria, BC, Canada V9E 2E7.

Department of Physics and Astronomy, Washington State University, Pullman, WA.

${ }^{4}$ European Southern Observatory, Karl-Schwarzschild-Strasse 2, 85748, Garching, Germany.

${ }^{5}$ GEPI, Observatoire de Paris, Section de Meudon, Meudon Cedex, France.

${ }^{6}$ Department of Physics and Astronomy, The Johns Hopkins University, Baltimore, MD 21218.

${ }^{7}$ Department of Astronomy and Astrophysics, University of California, Santa Cruz, CA 95064.

${ }^{8}$ See http://astro.berkeley.edu/ bait/kait.html.
}

The spectral and temporal properties of this object resemble those of M31-RV (discovered by Rich et al. 1989; see, e.g., Mould et al. 1990; Bryan \& Royer 1992), V838 Mon (discovered by Brown 2002; see, e.g., Kimeswenger et al. 2002; Bond et al. 2003; Corradi \& Munari 2007), and possibly the less studied object V4332 Sgr (Martini et al. 1999). However, the M85 transient is the most luminous member of the V838 Mon class. The favored model for this emerging class of V838 Mon-like objects (also known as luminous red novae ${ }^{9}$ ) is that they are the result of stellar mergers (e.g., Soker \& Tylenda 2006). However, other models have been suggested to explain these objects (e.g., Retter \& Marom 2003; Lawlor 2005). The nature of these events, with their energetics lying between the realms of supernovae and novae, remains uncertain.

In this paper we present Hubble Space Telescope (HST) Advanced Camera for Surveys (ACS)/Wide Field Camera (WFC) and Near Infrared Camera and Multi-Object Spectrometer (NICMOS) observations, as well as Palomar $5 \mathrm{~m}$ spectroscopy, of the environment of M85 OT 2006-1. The observations are used to characterize the environment of the transient and to set a limit on the mass of the progenitor.

\section{OBSERVATIONS}

M85 was observed using HST ACS in 2003 as part of the HST ACS Virgo Cluster Survey (Côté et al. 2004). Subsequently, the transient was observed serendipitously with ACS/WFC and NICMOS/NIC2 in 2006 (GO-10515) as a follow-up study to Peng et al. (2006). The ACS observations on 2006 were obtained 18 days after the discovery of the transient. The log of observations, the measured magnitude of the M85 transient, or the $\sim 3 \sigma$ upper limit at the OT location, as derived from the HST images taken on 2003 and 2006, are listed in Table 1. The HST images of the galaxy and the transient environment are presented in Figures 1 and 2.

9 This term was introduced by Kulkarni et al. 2007. 
TABLE 1

Photometry and Limiting Magnitude

\begin{tabular}{|c|c|c|c|c|c|}
\hline \multirow[b]{2}{*}{ Date } & \multirow{2}{*}{$\begin{array}{c}\text { EXPOSURE } \\
\text { (s) }\end{array}$} & \multirow[b]{2}{*}{ BAND } & \multirow[b]{2}{*}{ MAGNITUDE $^{\mathrm{a}}$} & \multicolumn{2}{|c|}{ Limiting Magnitude } \\
\hline & & & & Apparent & Absolute \\
\hline 2003 Feb $1 \ldots \ldots$. & 750 & $\mathrm{~F} 475 \mathrm{~W}(g)$ & $\ldots$ & $>26.9$ & $>-4.5$ \\
\hline 2003 Feb $1 \ldots \ldots$. & 1120 & $\mathrm{~F} 850 \mathrm{LP}(z)$ & $\ldots$ & $>25.1$ & $>-6.2$ \\
\hline 2006 Jan $24 \ldots \ldots$ & 2204 & $\mathrm{~F} 475 \mathrm{~W}(g)$ & 20.57 & & \\
\hline 2006 Jan $24 \ldots \ldots$ & 2224 & $\mathrm{~F} 814 \mathrm{~W}(i)$ & 18.62 & $>25.3$ & $>-6.0$ \\
\hline 2006 Feb $28 \ldots .$. & 500 & $\mathrm{~F} 160 \mathrm{~W}(H)$ & 17.82 & $>21.2$ & $>-10.1$ \\
\hline
\end{tabular}

${ }^{a}$ Vega-based magnitude corrected for infinite aperture (Sirianni et al. 2005). Errors in photometry are about 0.02 mag for the ACS observations, and 0.05 mag for the NICMOS observations. The NICMOS magnitude is calibrated using 2MASS stars in the field of view.

$\mathrm{b}$ Vega-based limiting magnitude as estimated by adding artificial point sources to the images in the neighborhood of the transient and inspection of the images for the added sources. The absolute magnitudes are calculated assuming a distance of 17.8 Mpc to M85 (Mei et al. 2007) and Galactic extinction of $E_{B-V}=0.031$ (Schlegel et al. 1998; Cardelli et al. 1989). Note, however, that distance estimates to M85 range between $14 \mathrm{Mpc}$ (Ciardullo et al. 2002) to $18.6 \mathrm{Mpc}$ (Blakeslee et al. 2001).

On 2007 January 20, after the M85 OT 2006-1 faded away, we obtained a spectrum at the location of M85 OT 2006-1. The spectrum (Fig. 3 ) consist of $4 \times 300$ s exposures with the double beam spectrograph mounted on the Palomar $5 \mathrm{~m}$ telescope. We used the 600 lines $\mathrm{mm}^{-1}$ grating blazed at $9500 \AA$ in the red arm. The $2^{\prime \prime}$ slit was centered on the nucleus of M85, at a position angle of $185^{\circ}$. The position angle was chosen such that the location of the transient will be included in the slit. On 2005 June 25, M85 was observed by Spitzer with the Multiband Imaging Photometer for Spitzer (MIPS). The $70 \mu \mathrm{m}$ image, with exposure time of $670 \mathrm{~s}$, is shown in Figure 4.

\section{RESULTS}

V838 Mon-like objects are found in both young regions (e.g., V838 Mon; Afșar \& Bond 2007) and old stellar populations (e.g., M31 RV; Bond \& Siegel 2006). M85 OT 2006-1 took place in an early-type galaxy. Therefore, as we explain below, it can be used to set an upper limit on the minimal progenitor mass that can produce V838 Mon-like eruptions.

From the two-dimensional spectrum, shown in Figure 3, we can set an upper limit on the flux of the $\mathrm{H} \alpha$ emission at the location of M85 OT 2006-1 of $<6 \times 10^{-14} \mathrm{ergs} \mathrm{cm}^{-2} \mathrm{~s}^{-1}$, at the $3.5 \sigma$ level (equivalent to luminosity of $1.6 \times 10^{39} \mathrm{ergs} \mathrm{s}^{-1}$ ). This corresponds to a star formation rate smaller than about $10^{-2} M_{\odot} \mathrm{yr}^{-1}$ (Kennicutt 1998) in a radius of $\sim 100 \mathrm{pc}$ around the transient location. For comparison, the $\mathrm{H} \alpha$ luminosity of the Orion nebula is about $\sim 10^{41} \mathrm{ergs} \mathrm{s}^{-1}$ (Haffner et al. 2003, assuming a distance of 392 pc; Jeffries 2007). Therefore, our observations rule out the presence of a prominent star-forming region in this location. Moreover, based on the far-IR flux in the region of the transient, obtained from the Spitzer MIPS $70 \mu \mathrm{m}$ image shown in Figure 4, we can set an upper limit on the star formation rate in this region to be less than $10^{-5} M_{\odot} \mathrm{yr}^{-1}$ (Kennicutt 1998).

The absence of $\mathrm{H}$ II regions in M85 rule out the possibility that the progenitor had a delay (from birth to outburst) of $<10 \mathrm{Myr}$ (corresponds to $\gtrsim 40 M_{\odot}$ ), which is the typical lifetime of $\mathrm{H}$ II regions (e.g., Mayya 1995).

An independent limit on the age and mass of the progenitor can be inferred from the absence of stars brighter than $z$-band absolute magnitude $M_{z}=-6.2$ in the transient environment. From the Lejeune \& Schaerer (2001) stellar tracks, we find that stars older than $50 \mathrm{Myr}$ (and therefore, more massive than $7 M_{\odot}$ ) do not reach $z$-band absolute magnitudes brighter than $M_{z}=$ $-6.2 \mathrm{mag}$ (at the red supergiant stage). We note that the $z$-band stellar track magnitudes were obtained by interpolation of the $I$ and $J$ bands. Therefore, we can set a lower limit on the age of the most massive stars in the transient environment to be $\gtrsim 50 \mathrm{Myr}$, which corresponds to mass $<7 M_{\odot}$ (assuming solar metallicity). Otherwise, we were likely to detect individual stars in this region. We note that we can limit the extinction in the transient location to $A_{i}<0.8 \mathrm{mag}$, based on the OT Balmer lines ratio, assuming case-B recombination (Kulkarni et al. 2007).

Ferrarese et al. (2006) reported possible faint wisps and patches of dust in M85. Moreover, Schweizer \& Seitzer (1992) reported

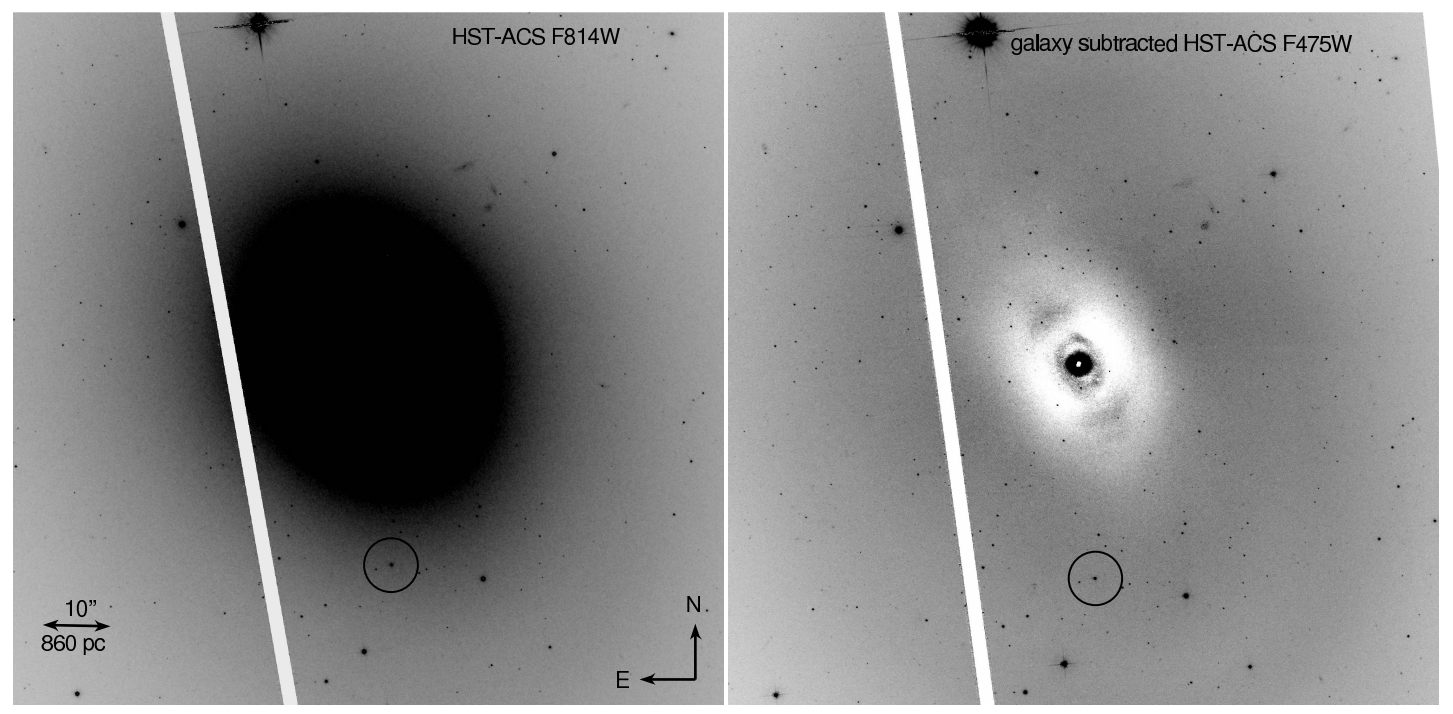

FIG. 1.-Left: HST ACS F814W-band image of the galaxy M85, obtained on 2006 January 24 (18 days after the discovery). The transient, which is well detected, is

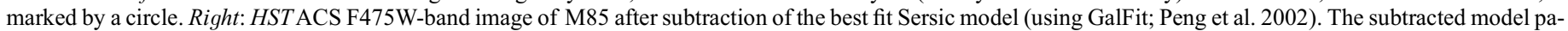

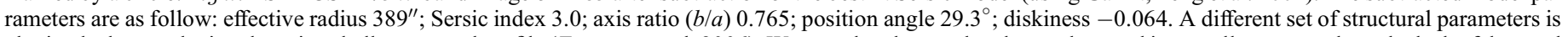

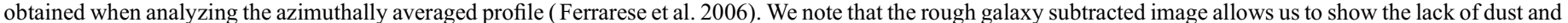

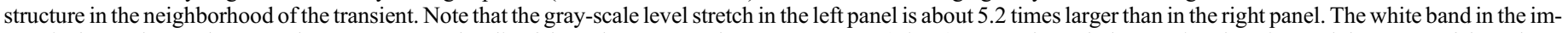

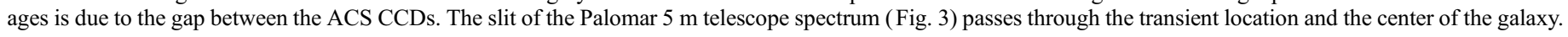



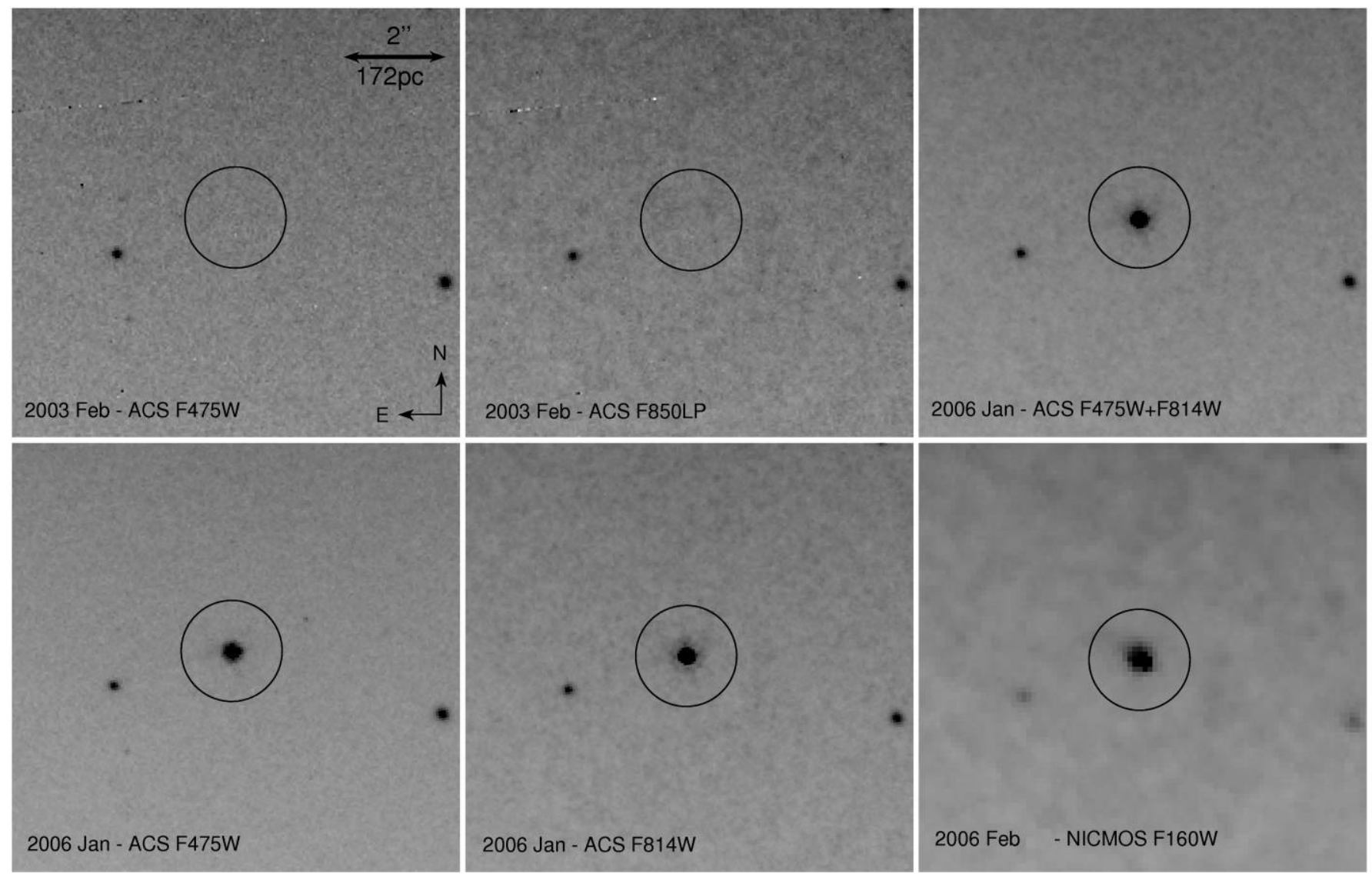

FIg. 2.-Zoom-in on the environment of M85 OT 2006-1 HST ACS and NICMOS/NIC2 images. The circle, with radius of $1^{\prime \prime}$, marks the position of the transient. Note that the F475W + F814W is a sum of the F475W and the F814W images.

that M85 is somewhat bluer than typical S0 galaxies and therefore possibly younger. This claim is supported by Terlevich \& Forbes (2002), who estimated the age and metallicity based on line indices. They have found a mean luminosity-weighted age of $1.6 \pm 0.3 \mathrm{Gyr}$ and metallicity of $[\mathrm{Fe} / \mathrm{H}]=0.44$ and $[\mathrm{Mg} / \mathrm{Fe}]=$ 0.08 . We note that the actual mean age is probably higher than that indicated by line indices given that younger populations have higher weight than old population. If the mean age is representative of the progenitor of M85 OT 2006-1, then we can set a lower limit of about 1 Gyr on the age of M85 OT 2006-1 progenitor(s). This further suggests that the mass of the progenitor/s is probably below $2 M_{\odot}$ (the lifetime of solar metallicity $>2 M_{\odot}$ stars is $<1$ Gyr; Lejeune \& Schaerer 2001). This limit is based on the mean stellar age of this galaxy. However, stars younger than $1 \mathrm{Gyr}$ may be present in this galaxy in relatively small numbers.

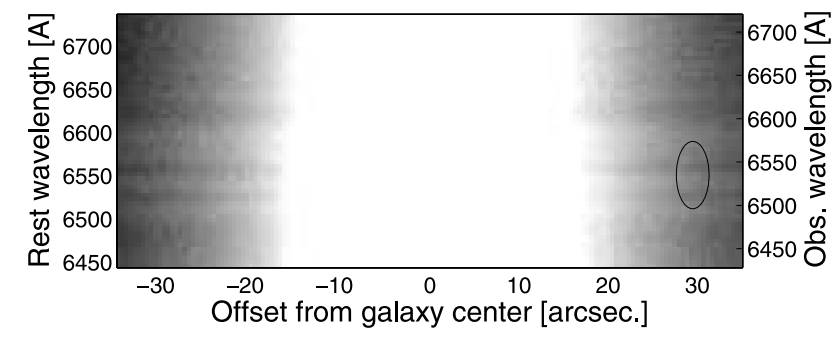

FIG. 3.-Two-dimensional spectrum of M85 and the transient environment $\left(+30^{\prime \prime}\right.$ offset from the galaxy center along the slit), obtained about 1 year after the transient discovery, covering the $\mathrm{H} \alpha$ wavelength region (ellipse). No $\mathrm{H} \alpha$ emission is seen in the vicinity of the transient. The spectrum is shown before sky subtraction.

\section{DISCUSSION}

Although several models exist for V838 Mon-like objects (e.g., Soker \& Tylenda 2003; Lawlor 2005), in the absence of detailed simulations, the nature of these objects remain elusive. A clue to their origin can be derived from their environment, luminosity function, and rate. Given that only a small number of these objects are known, and they were found serendipitously in various searches, the luminosity function and rate are not well constrained. However, the fact that at least two events were observed in our Galaxy (i.e., V838 Mon and V4332 Sgr) in the last

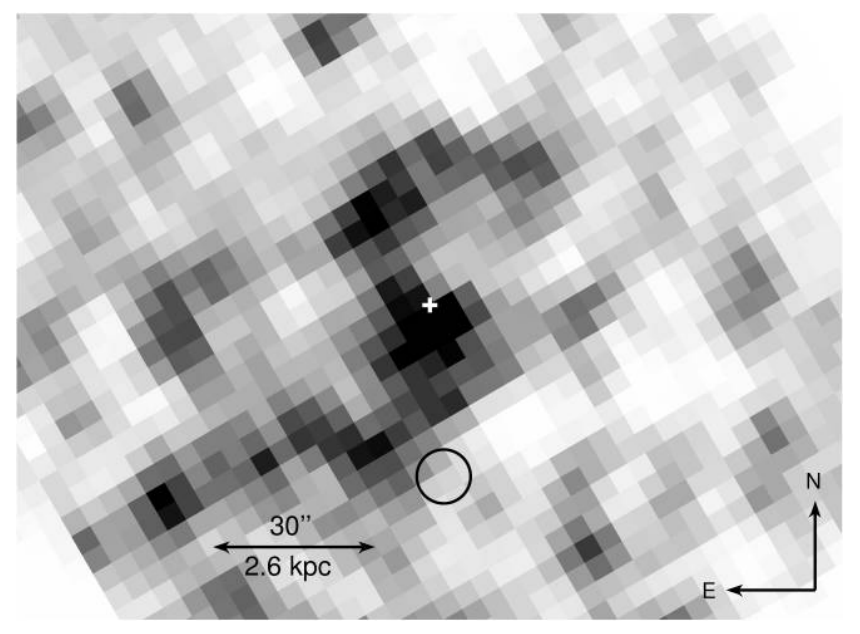

FIG. 4.-Spizter MIPS $70 \mu \mathrm{m}$ image of M85. The plus sign marks the visiblelight center of the galaxy, while the circle marks the position of the transient. 
$\sim 13$ years suggests that they have a higher rate than SNe. We can set a lower limit on their rate, of $0.019 \mathrm{yr}^{-1} L_{\mathrm{MW}}^{-1}$, at the $95 \%$ confidence level, where $L_{\mathrm{MW}}$ is the Milky Way luminosity.

Now we discuss the implications of our observations for a specific model for V838 Mon-like objects. Soker \& Tylenda (2006) presented a model for violent stellar mergers in which, prior to the merger, the spins and orbital frequencies of the binary star are losing synchronization due to the Darwin instability (e.g., Eggleton \& Kiseleva-Eggleton 2001). They found that for a given primary mass, the maximal energy production obtained for a binary mass ratio of $\sim 1 / 50$, is $\sim 2.5 \times 10^{-3} G M_{1}^{2} / R_{1}$, where $G$ is the gravitational constant, and $M_{1}$ and $R_{1}$ are the mass and radius of the primary star. Given the upper limit on the progenitor mass, based on the mean stellar age in M85, $<2 M_{\odot}$, and assuming a main-sequence mass-radius relation, $R \propto M^{0.7}$, the maximum available energy in their model is short by a factor of three in the total energy production, as compared to the radiated energy of M85 OT 2006- 1 in the first 2 months, $\sim 8 \times 10^{46}$ ergs (assuming a distance of $17.8 \mathrm{Mpc}$ to M85; Mei et al. 2007). Moreover, it is expected that a large fraction of the energy will go into lifting the outer region of the star rather than radiated away. Furthermore, if the primary is an evolved star, then its radius will be larger than the radius of a main-sequence star with the same mass, and the extracted energy will be even smaller. This suggests that either more detailed modeling of violent stellar mergers is required, or that this event is not the result of a violent stellar merger. Another possible solution is that the mass of the progenitor is somewhat larger. A larger progenitor mass will still be consistent with our upper limit of $7 M_{\odot}$, which is based on the absence of stars brighter than $I \sim-6$ mag. For example, according to Soker $\&$ Tylenda (2006) model, a $7 M_{\odot}$ progenitor can yield $\sim 4$ times more energy than a $2 M_{\odot}$ progenitor and may explain the discrepancy. We note, however, that other kinds of instabilities can lead to stellar mergers (e.g., in triple systems) and that the above comparison is valid only for the specific case discussed by Soker \& Tylenda (2006).

Existing hydrodynamical simulations of the common envelope phase in stellar mergers (and also star + neutron star mergers) predict that the total dissipated energy is of the order of that observed in V838 Mon and M85 OT 2006-1 (e.g., Taam \& Bodenheimer 1989; Terman et al. 1995; Terman \& Taam 1996). Moreover, simulations of the common envelope phase predicts that most of the envelope will be ejected in the equatorial plane (e.g., Taam \& Ricker 2006). Indeed, in Rau et al. (2007) we reported evidence suggesting that the expansion of M85 OT 2006-1 is asymmetric. However, more detailed hydrodynamical simulations of the vast parameter space available for stellar mergers are needed in order to understand these processes and to test if V838 Mon-like objects are indeed the results of stellar mergers.

To summarize, we show that, in contrast to V838 Mon, but similarly to M31 RV, M85 OT 2006-1 was probably produced by members of an old stellar population ( $>1 \mathrm{Gyr})$, and that its progenitor/s mass was probably $\leqslant 2 M_{\odot}$. These constraints narrow down the allowed venue of stellar models for the nature of this event.

This work is supported in part by grants from NSF and NASA.
Afșar, M., \& Bond, H. E. 2007, AJ, 133, 387

Blakeslee, J. P., Lucey, J. R., Barris, B. J., Hudson, M. J., \& Tonry, J. L. 2001, MNRAS, 327, 1004

Bond, H. E., \& Siegel, M. H. 2006, AJ, 131, 984

Bond, H. E., et al. 2003, Nature, 422, 405

Brown, N. J. 2002, IAU Circ., 7785, 1

Bryan, J., \& Royer, R. E. 1992, PASP, 104, 179

Cardelli, J. A., Clayton, G. C., \& Mathis, J. S. 1989, ApJ, 345, 245

Ciardullo, R., Feldmeier, J. J., Jacoby, G. H., Kuzio de Naray, R., Laychak, M. B., \& Durrell, P. R. 2002, ApJ, 577, 31

Corradi, R. L. M., \& Munari, U. 2007, in ASP Conf. Ser. 363, The Nature of V838 Mon and Its Light Echo, ed. R. L. M. Corradi \& U. Munari (San Francisco: ASP), 363

Côté, P., et al. 2004, ApJS, 153, 223

Eggleton, P. P., \& Kiseleva-Eggleton, L. 2001, ApJ, 562, 1012

Ferrarese, L., et al. 2006, ApJS, 164, 334

Filippenko, A. V., Li, W. D., Treffers, R. R., \& Modjaz, M. 2001, in IAU Colloq. 183, Small Telescope Astronomy on Global Scales, ed. B. Paczyński, W.-P. Chen, \& C. Lemme (ASP Conf. Ser. 246; San Francisco: ASP), 121

Haffner, L. M., Reynolds, R. J., Tufte, S. L., Madsen, G. J., Jaehnig, K. P., \& Percival, J. W. 2003, ApJS, 149, 405

Jeffries, R. D. 2007, MNRAS, 376, 1109

Kennicutt, R. C., Jr. 1998, ApJ, 498, 541

Kimeswenger, S., Lederle, C., Schmeja, S., \& Armsdorfer, B. 2002, MNRAS, 336, L43

\section{REFERENCES}

Kulkarni, S. R., 2007 Nature, 447, 458

Lawlor, T. M. 2005, MNRAS, 361, 695

Lejeune, T., \& Schaerer, D. 2001, A\&A, 366, 538

Martini, P., Wagner, R. M., Tomaney, A., Rich, R. M., della Valle, M., \& Hauschildt, P. H. 1999, AJ, 118, 1034

Mayya, Y. D. 1995, AJ, 109, 2503

Mei, S., et al. 2007, ApJ, 655, 144

Mould, J., et al. 1990, ApJ, 353, L35

Peng, C. Y., Ho, L. C., Impey, C. D., \& Rix, H.-W. 2002, AJ, 124, 266

Peng, E. W., et al. 2006, ApJ, 639, 838

Rau, A., Kulkarni, S. R., Ofek, E. O., \& Yan, L. 2007, ApJ, 659, 1536

Retter, A., \& Marom, A. 2003, MNRAS, 345, L25

Rich, R. M., Mould, J., Picard, A., Frogel, J. A., \& Davies, R. 1989, ApJ, 341, L51

Schlegel, D. J., Finkbeiner, D. P., \& Davis, M. 1998, ApJ, 500, 525

Schweizer, F., \& Seitzer, P. 1992, AJ, 104, 1039

Sirianni, M., et al. 2005, PASP, 117, 1049

Soker, N., \& Tylenda, R. 2003, ApJ, 582, L105 2006, MNRAS, 373, 733

Taam, R. E., \& Bodenheimer, P. 1989, ApJ, 337, 849

Taam, R. E., \& Ricker, P. M. 2006, preprint (astro-ph/0611043)

Terlevich, A. I., \& Forbes, D. A. 2002, MNRAS, 330, 547

Terman, J. L., \& Taam, R. E. 1996, ApJ, 458, 692

Terman, J. L., Taam, R. E., \& Hernquist, L. 1995, ApJ, 445, 367 